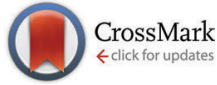

Cite this: Phys. Chem. Chem. Phys., 2014, 16, 26887

Received 14th August 2014, Accepted 28th October 2014

DOI: $10.1039 / c 4 c p 03634 d$

www.rsc.org/pccp

\title{
On the origin of fluorescence emission in optically non-linear DCNP crystals $\uparrow$
}

\author{
O. Morawski, ${ }^{a}$ A. L. Sobolewski, ${ }^{a}$ B. Kozankiewicz, ${ }^{a}$ L. Sznitko ${ }^{b}$ and A. Miniewicz ${ }^{\star b}$
}

We study absorption and emission spectra of optically nonlinear single crystals of 3-(1,1-dicyanoethenyl)1-phenyl-4,5-dihydro- $1 \mathrm{H}$-pyrazole (DCNP) at $5 \mathrm{~K}$. We argue that fluorescence has a complex origin, it is emitted from the excitonic band, with the bottom at $\sim 18115 \mathrm{~cm}^{-1}$, and from trap states, and the two main traps have depths of $\sim 875$ and $\sim 2465 \mathrm{~cm}^{-1}$. The excitonic origin of the emission is confirmed by the vibrational structure of fluorescence, closely resembling vibrations observed in the Raman scattering spectrum (recorded for DCNP crystals at 295 K) and by very short decay time of the excitonic emission, as a consequence of exciton migration and trapping at deep traps.

\section{Introduction}

Pyrazoles are important heterocyclic compounds whose derivatives are used in a number of applications in medicine. ${ }^{1-3}$ This, so called azole family, includes also imidazoles, oxadiazoles, oxazoles and thiazoles. ${ }^{4}$ Pyrazoles, with their ring structure composed of three carbon and two nitrogen atoms, create the $\pi$-electron conjugated systems that lead to the possibility of molecular nonlinear optical (NLO) activity. Optical and NLO properties of a number of pyrazole derivatives were already reported in the literature. ${ }^{5-14}$

The title compound 3-(1,1-dicyanoethenyl)-1-phenyl-4,5-dihydro$1 H$-pyrazole, abbreviated as DCNP $\left(\mathrm{C}_{13} \mathrm{H}_{10} \mathrm{~N}_{4}, M_{\mathrm{w}}=222.25 \mathrm{~g}\right)$, is a good example of a low-molecular mass organic dye classified as a nonlinear optical chromophore due to its anisotropic "push-pull" character as described in ref. 12. The molecular structure of this compound has been schematically shown in Fig. 1. Fluorescence properties of the single crystals of DCNP under single and twophoton excitation conditions at temperatures between room and $77 \mathrm{~K}$ were already reported in ref. 14 . Within this temperature range the fluorescence spectrum is composed of two broad bands, one centred at about 580 and the second at $640 \mathrm{~nm}$, with continuous transfer of the intensity from the higher energy band to the lower energy band following a temperature increase. Such an observation raises the question about the origin of both bands. The present studies should also help in interpretation of luminescence from

\footnotetext{
${ }^{a}$ Institute of Physics, Polish Academy of Sciences, PL-02668 Warsaw, Poland

${ }^{b}$ Institute of Physical and Theoretical Chemistry, Department of Chemistry,

Wroclaw University of Technology, Wybrzeze Wyspianskiego 27,

PL-50370Wroclaw, Poland.E-mail: andrzej.miniewicz@pwr.edu.pl

$\dagger$ Electronic supplementary information (ESI) is available: Spectra of DCNP in the $n$-nonane Shpol'skii matrix; Raman spectra of DCNP crystals; and table with calculated and measured frequencies of vibrations of DCNP molecules and in a crystal. See DOI: 10.1039/c4cp03634d
}

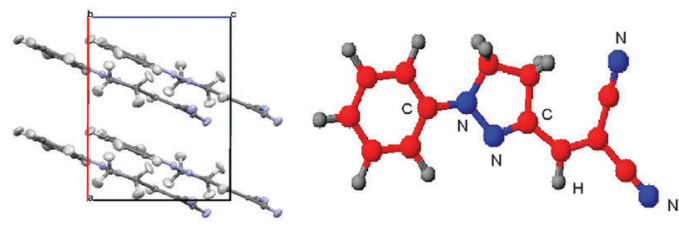

Fig. 1 Projection of molecular packing in the crystal viewed along the $b$-axis (after ref. 13) and the molecular structure of DCNP $\left(\mathrm{C}_{13} \mathrm{H}_{10} \mathrm{~N}_{4}\right)$.

DCNP nanocrystals and amplified spontaneous emission and lasing. ${ }^{15}$

DCNP crystallizes in the monoclinic non-centrosymmetric space group $C c$ (point group $\mathrm{m}$ ) with pseudo-orthogonal geometry where $Z=4$ molecules of DCNP occupy a unit cell. The first crystallographic structure of DCNP crystals was reported by Allen et al. ${ }^{12}$ The more precise structural studies of this compound both by X-ray and neutron diffraction have been done by Cole et al. ${ }^{13}$ at $90,100,200$ and $290 \mathrm{~K}$. The cell parameters $a=11.8751 \AA, b=12.3735 \AA, c=7.8876 \AA, \beta=90.412$, and $Z=4$ at $290 \mathrm{~K}$ have been measured by XRD. ${ }^{13}$ No phase transition was reported in this crystal down to $90 \mathrm{~K}$. The densities at $290 \mathrm{~K}$ and $100 \mathrm{~K}$ as calculated from the unit cell parameters are 1.274 and $1.321 \mathrm{Mg} \mathrm{m}^{-3}$, respectively. The molecules are well aligned unidirectionally. From neutron diffraction studies ${ }^{13}$ it follows that the structure is stabilized by the presence of two weak intermolecular $\mathrm{C}-\mathrm{H} \cdots \mathrm{N}$ hydrogen bonds. Parallel and head-to-tail arrangement of the molecules in the crystallographic structure, such that the nitrile group in one molecule is adjacent to the phenyl group in the next molecule, is directly responsible for the extremely large electro-optic coefficient of DCNP crystals, represented by the dominating second order susceptibility term $\chi_{333}^{(2)}(-\omega ; \omega, 0)=$ $-2.3 \times 10^{-9} \mathrm{~m} \mathrm{~V}^{-1},{ }^{12}$ where the subscript 3 denotes the $z$-axis of the crystal that is nearly parallel to the long molecular axis. 
Polarization of DCNP crystals at $290 \mathrm{~K}$, calculated by considering four molecules with the ground state dipole moment $\mu=7.5 D=2.502 \times 10^{-29} \mathrm{C} \mathrm{m}$ placed in a unit cell of the volume $V=1158.95 \AA^{3}$ and oriented in the same direction, is equal to $|P|=0.116 \mathrm{C} \mathrm{m}^{-2}$. Recently, we confirmed that single crystals of DCNP indeed show a pyroelectric effect in the $z$-direction (unpublished results). Infrared (1064 nm) $5 \mathrm{~ns}$ pulses emitted by an Nd:YAG laser induced in a DCNP crystal an efficient fluorescence due to the two photon absorption process or the reabsorption of second order harmonic generation (SHG) light. ${ }^{14,15}$ Molecules of DCNP when dispersed in the polymer films of polyetherketone or PMMA, oriented by a high dc electric field, i.e. by a corona poling process, also show the efficient SHG phenomenon. ${ }^{16-18}$

The common property of the majority of molecular crystals, even those of the best quality, is the presence of trap states, which may be due to structural faults, vacancies, or chemical impurities. Particularly, for the DCNP we believe that the traps are of structural origin. The starting material was very carefully purified. Moreover, from the work of Black et $a .^{19}$ it is known that for crystals grown from toluene solvent one deals with different growth rates along the polar axis of DCNP. Toluene appears to be selectively adsorbed at the faster growing polar end of the crystal (the cyano-rich direction) resulting in minor anisotropic misorientations being present in these crystals. ${ }^{19}$ This may point out that the main source of trapping states in DCNP single crystals is related to the presence of structural and not chemical traps.

Excitonic energy migration within the crystal, especially under ambient conditions, may result in efficient population of these trap states (as well as thermal depopulation of traps), therefore optical properties of a studied crystal may be disturbed by the traps. Thus, a deep understanding of the optical properties of a molecular crystal may require clarification of the origin of traps.

The first step into this direction is to determine trap's depths with respect to the crystal excitonic band. The purpose of the present work is to get experimental information about the energy location of the excitonic band in the single crystal of DCNP, which is necessary for determination of trap's depths. This goal is realized by studying absorption and fluorescence spectra of the crystal at $5 \mathrm{~K}$. In order to convince ourselves that we are investigating the properties of the bulk crystal we compared the vibronic structure of fluorescence (recorded at $5 \mathrm{~K}$ ) with Raman spectra (recorded at $295 \mathrm{~K}$ ) of a DCNP crystal. We also analysed the vibronic structure of fluorescence and fluorescence excitation spectra of isolated DCNP molecules dispersed in a Shpol'skii matrix of $n$-nonane at $5 \mathrm{~K}$. The spectral analysis is supported by the kinetic study.

\section{Results and discussion}

\subsection{Absorption and fluorescence of DCNP at 5 K}

Absorption and fluorescence spectra of the single crystal of DCNP recorded at $5 \mathrm{~K}$ are presented in Fig. 2. A sharp absorption edge, which can be identified at the bottom of an excitonic band, is observed at $\sim 552 \mathrm{~nm}\left(18115 \mathrm{~cm}^{-1}\right)$. However, the absorption of nonpolarized light at this wavelength does not drop to the

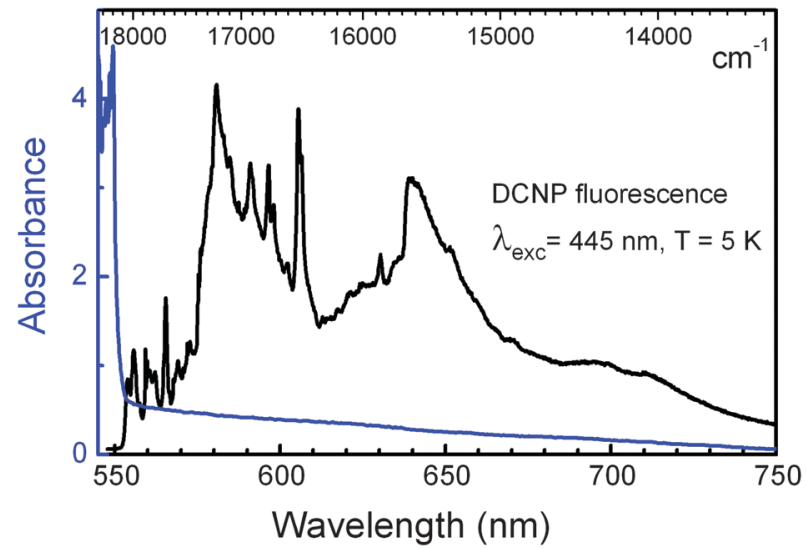

Fig. 2 Absorption (blue line) and fluorescence (black line) spectra of a $\sim 2.5 \mathrm{~mm}$ thick single crystal of DCNP recorded at $5 \mathrm{~K}$. No correction of absorbance due to light reflection from the sample surface has been applied.

zero value, but to the absorbance of about 0.6 (in a $\sim 2.5 \mathrm{~mm}$ thick crystal this corresponds to an average absorption coefficient of $\alpha=5.5 \mathrm{~cm}^{-1}$ ). Absorbance continues its slow decrease to lower values toward longer wavelengths over the whole spectral range presented in Fig. 2. Such behaviour indicates a contribution to the absorption from a manifold of broad, continuously deeper and deeper trap states. The residual absorbance (attributed to the trap states) is strongly dependent on light polarization (presented in Fig. S2 of ESI $\dagger$ ) - with the light polarized parallel to the long axis of DCNP molecule absorbance is considerably bigger $\alpha_{z}(633 \mathrm{~nm}) \approx 11 \mathrm{~cm}^{-1}$ than that with the light of perpendicular polarization amounting to $\alpha_{x}(633 \mathrm{~nm}) \approx 2 \mathrm{~cm}^{-1}$.

This observation is a signature of the presence of small density of absorbing species whose transition dipole moment is parallel to the long axis of DCNP molecules, e.g. molecules or their clusters with reversed dipole moments with respect to the majority of molecules. Typical absorption coefficients for the molecular crystal above absorption edge are of the order of $10^{3}-10^{4} \mathrm{~cm}^{-1}$. Our results ( $c f$. Fig. 2) are consistent with the absorption spectrum recorded at room temperature by Allen et $a l^{12}$ These authors observed a sharp absorption edge at $580 \mathrm{~nm}$ at room temperature, which is about $875 \mathrm{~cm}^{-1}$ lower in energy as compared with our absorption edge observed at $5 \mathrm{~K}$. They also observed residual absorption at longer wavelengths, polarized (like in our study) along the long axis of DCNP molecules. "Red-shift" of the absorption edge between that measured at $5 \mathrm{~K}$ and at room temperature may be attributed to "hot absorption", being the result of higher population of molecular vibrations in a crystal with a temperature increase. Another possible mechanism, acting in the same direction, is the change of the internal crystal energy with temperature: when temperature decreases the average crystal cohesive energy may increase, at the same time strengthening intermolecular interactions.

\subsection{Excitonic emission and trap states}

The fluorescence spectrum recorded at $5 \mathrm{~K}$ ( $c f$. Fig. 2) is composed of several relatively narrow bands (as compared with those observed at elevated temperatures ${ }^{14}$ ). The onset line of 


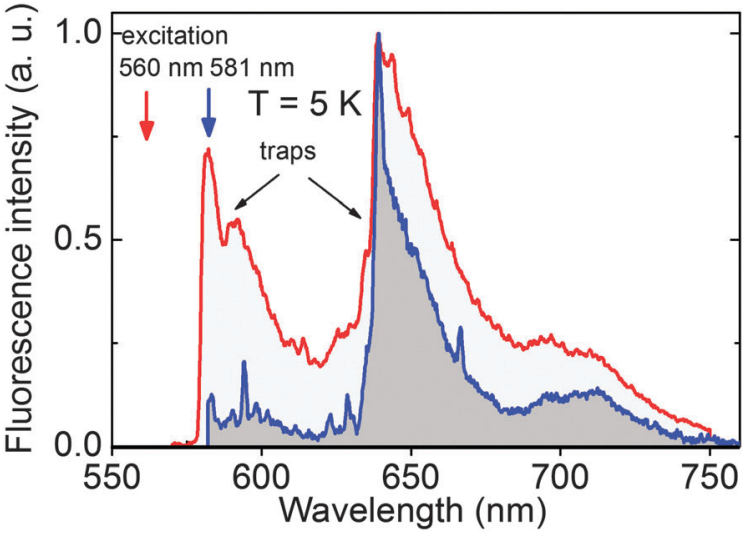

Fig. 3 Fluorescence spectra of DCNP crystals at $5 \mathrm{~K}$ obtained with the excitation wavelengths of 560 and $581 \mathrm{~nm}$, respectively.

fluorescence is located at about $552 \mathrm{~nm}$, i.e. it exactly matches the sharp drop of absorbance, confirming that at this wavelength we should locate energy at the bottom of the excitonic $S_{1}$ band of the DCNP crystal. The spectrum, which spans between 552 and $575 \mathrm{~nm}$ and excited with a $445 \mathrm{~nm}$ line, composes of several narrow lines. We attribute this part of the spectrum to excitonic emission, although we cannot exclude that the emission originates from very shallow traps of the X-trap origin. ${ }^{20}$ Broad bands with the onset at about 580 and $639 \mathrm{~nm}$ attribute to the trap states, with the depths of $\sim 875$ and $\sim 2465 \mathrm{~cm}^{-1}$, respectively. Such a conclusion is confirmed by the spectra presented in Fig. 3, which were obtained using the two different excitation wavelengths of 560 and $581 \mathrm{~nm}$. Under these conditions (excitation below the excitonic band) the trap states are excited directly. It is worth noting that shifting of excitation wavelength by only $1 \mathrm{~nm}$, i.e. from $580 \mathrm{~nm}$ to $581 \mathrm{~nm}$, results in fluorescence vanishing from the higher energy traps. Let us recall that the fluorescence emission from these two trap states (with the maxima at 580 and $639 \mathrm{~nm}$ ) was already reported at elevated temperatures. ${ }^{14}$

In the forthcoming presentation and discussion we concentrate on the excitonic emission (spread in the fluorescence spectrum between 552 and $575 \mathrm{~nm}$ ). In order to confirm the excitonic origin of this emission we collected fluorescence at $5 \mathrm{~K}$ in two different detection configurations. We focused an excitation beam of the $445 \mathrm{~nm}$ light (excitation to the excitonic band) on the crystal surface to a spot of diameter of about $0.5 \mathrm{~mm}$ and collected emission light from this spot and next we shifted the detection spot $1 \mathrm{~mm}$ apart from the excitation spot. The results of such an experiment are presented in Fig. 4. It is clearly seen that the spectrum "off-spot" lacks the excitonic component and is composed of emission from trap states only.

\subsection{Fluorescence decays at $5 \mathrm{~K}$}

The kinetic studies were performed using the $384 \mathrm{~nm}$ wavelength picosecond pulses (200 ps), which excited the crystal above the absorption edge, and we monitored fluorescence decays at different wavelengths. In Fig. 5 we show examples of normalized fluorescence decays measured in DCNP single

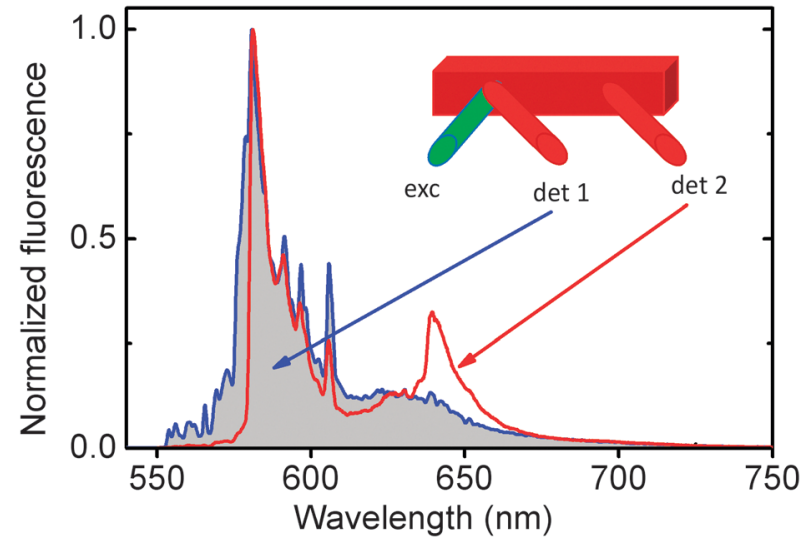

Fig. 4 Fluorescence spectra of DCNP single crystals recorded at $T=5 \mathrm{~K}$ "on-spot" (blue line, det 1) and "off-spot" $1 \mathrm{~mm}$ aside (red line, det 2). The DCNP crystal was excited by a $445 \mathrm{~nm}$ laser light; both spectra were normalized at maximum.

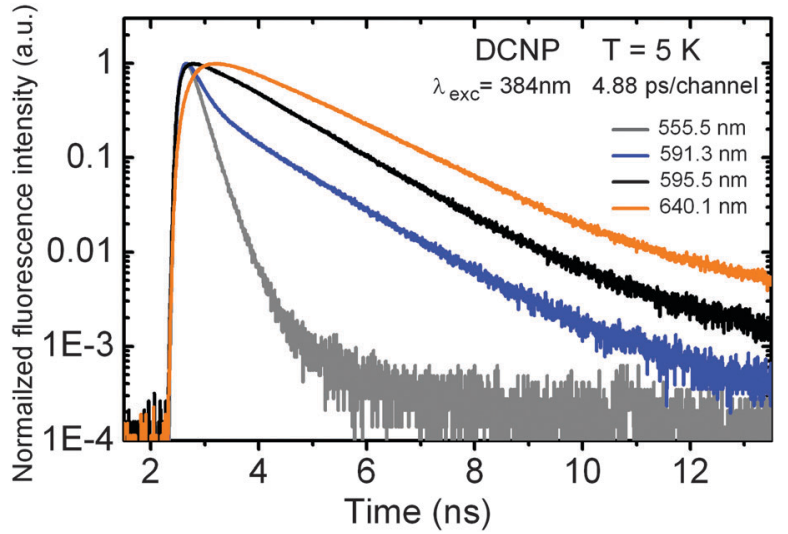

Fig. 5 Fluorescence decay profiles of DCNP single crystals at temperature $5 \mathrm{~K}$ excited with $384 \mathrm{~nm}$ picosecond laser pulses and measured at $555.5 \mathrm{~nm}$ (excitonic emission region), 591.3, 595.5 $\mathrm{nm}$ (a shallow trap at the $875 \mathrm{~cm}^{-1}$ emission region) and $640.1 \mathrm{~nm}$ (a deeper trap at the $2465 \mathrm{~cm}^{-1}$ emission region).

crystals at $T=5 \mathrm{~K}$ and excited above the absorption edge as a function of emission wavelength.

In the "blue" part of the fluorescence spectrum, corresponding to the excitonic emission, fluorescence decays were found to be very fast, on the subnanosecond time scale. Fitting the decays to mono-exponential dependence by using deconvolution of the signal we found decay times equal to 210 ps for the observation at $555.5 \mathrm{~nm}$ and $255 \mathrm{ps}$ at $564.8 \mathrm{~nm}$, respectively. Considerably longer and non-exponential decays were detected at wavelengths of observation longer than $575 \mathrm{~nm}$. Fitting the decay curves to bi-exponential dependence we got a short-lived component of about $1.3 \mathrm{~ns}$ and a long-lived component of about $5 \mathrm{~ns}$. These two values were practically unchanged over the whole measured long wavelength spectral range of 570-700 nm, but with lengthening of the observation wavelength the amplitude of the long-lived component was raising whereas that of the short-lived one was decreasing. Kinetics of fluorescence emission observed at $640.1 \mathrm{~nm}$ shows a curve with maximum shifted in time with respect to the 
excitation pulse position; this is manifestation of the process of excitonic diffusion. Fluorescence decays shown in Fig. 5 point out to a complex excitation energy migration toward the trapping species. Traps strongly contribute to the emission of DCNP crystals at longer wavelengths. The most meaningful result is the very fast decay of excitonic fluorescence indicating efficient migration of excitons already at $5 \mathrm{~K}$ and their trapping at deep (at this temperature) trap states.

\subsection{Vibrational structure of luminescence}

For many organic molecules the optical spectra of several aggregates show a vibrational structure principally similar to the internal vibrational modes of the monomers. ${ }^{21}$ This phenomenon is also observed for DCNP molecules, whose fluorescence spectrum of monomers in an alkane matrix shows the vibronic structure similar to that in the crystal phase. Thus the comparison of Raman and fluorescence spectra can be used to check if the trapping states of the crystal are of the DCNP or the chemical impurity origin.

In order to confirm that the fluorescence spectrum detected within the 552-575 $\mathrm{nm}$ range has the excitonic (bulk) origin we compare the "blue" part of the spectrum presented already in Fig. 2 with the Raman spectrum of a single DCNP crystal (collected at $295 \mathrm{~K}$ ). The result, where the spectra are compared
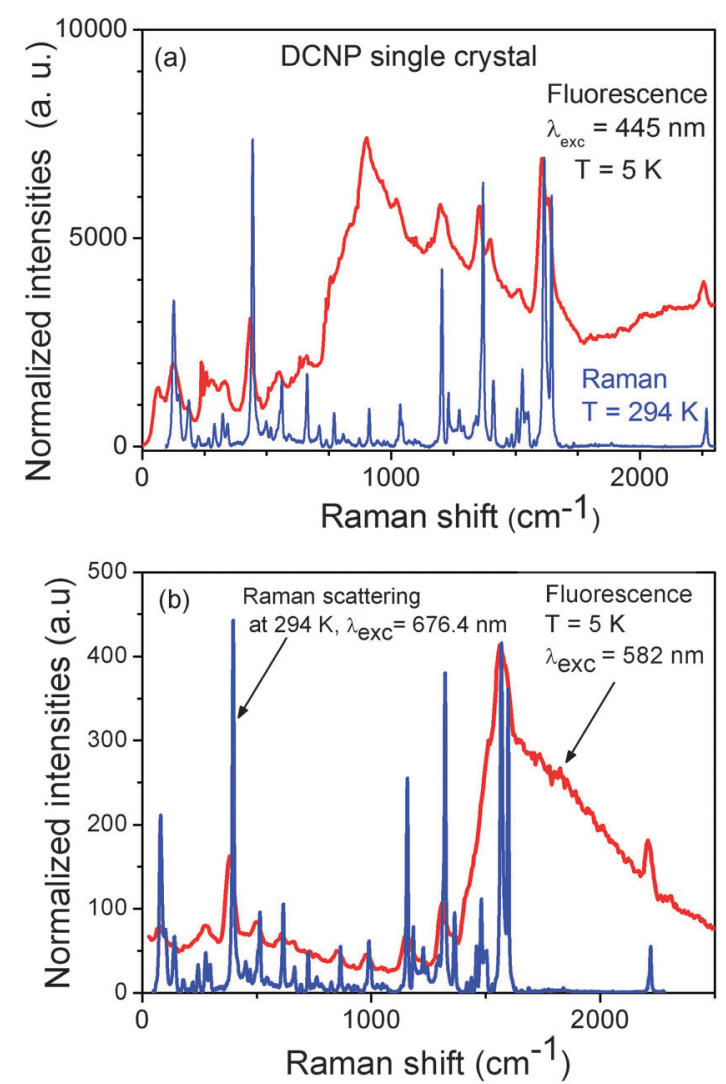

Fig. 6 Raman spectrum of a DCNP single crystal measured at $295 \mathrm{~K}$ $\left(\lambda_{\mathrm{R}}=676.4 \mathrm{~nm}\right.$, blue lines) superimposed on the low temperature, $T=5 \mathrm{~K}$, fluorescence spectra of the crystal (red lines) excited to the excitonic band by $445 \mathrm{~nm}$ laser light (a) and to the trap state absorbing at $582 \mathrm{~nm}$ (b). and plotted in a wavenumber scale, is shown in Fig. 6a. Agreement between both spectra is quite good - several lines in the fluorescence spectrum have their partners in the Raman spectrum. A broad band, which builds up above $700 \mathrm{~cm}^{-1}$ (corresponding in Fig. 2 to wavelengths longer than $575 \mathrm{~nm}$ ), is attributed to a deep trap state. Raman scattering reports on the molecular oscillation energies of the bulk crystal and is not sensitive to trap states (if the concentration of traps is low, like in DCNP crystals under study). The good matching of main Raman lines with the fluorescence fine structure clearly confirms the bulk origin of fluorescence emission in the considered spectral range (552-575 $\mathrm{nm})$.

The vibrational structure of the Raman spectrum can be further used to get information about the origin of a deep trap contributing to the fluorescence spectrum of DCNP crystals at wavelengths longer than $580 \mathrm{~nm}$. These traps, and deeper traps, are directly populated with the $582 \mathrm{~nm}$ excitation wavelength, as already shown in Fig. 3. Very good matching of both, Raman and fluorescence trap spectra is presented in Fig. 6b. This result convinced us that the trap as deep as $\sim 875 \mathrm{~cm}^{-1}$ below the excitonic band is also created on DCNP molecules, and not on chemical impurities. Fluorescence from impurities having different molecular structures than DCNP molecules should show fine structure different from that observed in the Raman scattering experiment.

\subsection{Shpol'skii spectra of DCNP at $5 \mathrm{~K}$}

Shpol'skii spectra of DCNP in $n$-nonane at $5 \mathrm{~K}$ provide information about the spectral characteristics of DCNP monomers. In this matrix DCNP molecules occupy two, well-defined, insertion sites, with the $(0,0)$ lines located at 499.6 and $502.5 \mathrm{~nm}(20012$ and $19905 \mathrm{~cm}^{-1}$, respectively), as presented in Fig. S1 of the ESI. $\dagger$ Thus, the onset of the DCNP crystal fluorescence is shifted to a lower energy, by at least, $\sim 1900 \mathrm{~cm}^{-1}$ with respect to that of isolated DCNP molecules. Vibrational frequencies of DCNP molecules embedded in both sites are similar. Slightly different frequencies of vibrations present in the fluorescence spectrum and in the fluorescence excitation spectrum reflect a geometry difference of DCNP molecules in the electronic ground and the lowest excited singlet states, respectively. Vibrations observed in the Raman spectrum of DCNP crystals superimposed on the fluorescence spectrum of DCNP molecules isolated in the $n$-nonane matrix (presented for site 1) reproduce most of the molecular vibrational lines ( $c f$. Fig. 7). Such a result is expected for a molecular crystal, where excitonic interactions between different molecules are considerably weaker than those forming a DCNP molecule.

Fluorescence decays for DCNP in a $n$-nonane matrix are mono-exponential and do not depend on the wavelength of observation as well as on temperature up to $180 \mathrm{~K}$, above this temperature the $n$-nonane melts. The fluorescence decay time for the monomer is $\tau_{\text {monomer }}=4.8 \mathrm{~ns}$, i.e. close to 3.7 and $5.6 \mathrm{~ns}$, already reported for DCNP crystals at $293 \mathrm{~K}^{14}$

Finally, let us mention that using a Parker type phosphorimeter, equipped with mechanical choppers and a sensitive EMCCD camera, we were not able to detect any long-lived (longer than $100 \mu \mathrm{s}$ ) phosphorescence emission in a DCNP crystal at $5 \mathrm{~K}$. We scanned the whole spectral range between 600 


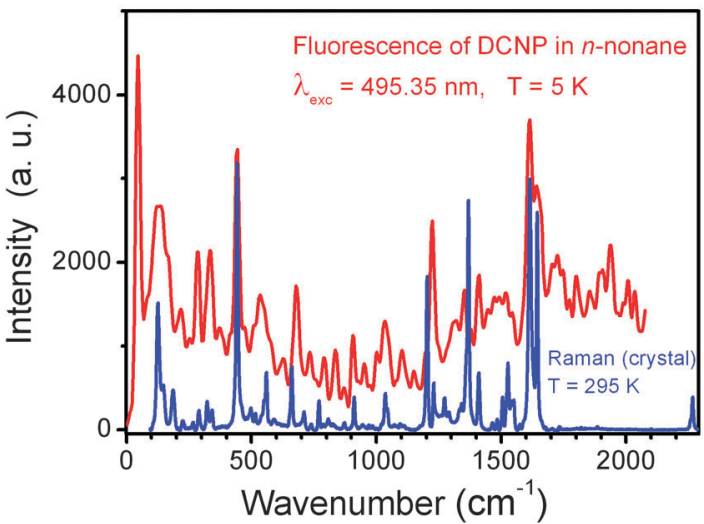

Fig. 7 Raman spectrum recorded for DCNP crystals (at $295 \mathrm{~K}, \lambda_{R}=676.4 \mathrm{~nm}$ ) superimposed on the fluorescence spectrum of DCNP molecules in a $n$-nonane matrix at $5 \mathrm{~K}$ excited by a $495.35 \mathrm{~nm}$ laser light (site 1).

and $1100 \mathrm{~nm}$. Even more, our efforts to enhance intersystem crossing efficiency in DCNP by dissolving this compound in iodoethane, which is known to increase population of the triplet states due to the external heavy atom effect, appeared unsuccessful - we were not able to monitor any phosphorescence. We may thus conclude that intersystem crossing from the excited singlet state $S_{1}$ to the triplet manifold in DCNP is not an efficient channel and excitation energy relaxation proceeds via radiative, fluorescence, and nonradiative, internal conversion channels.

\section{Experimental}

\subsection{Crystal preparation}

The DCNP compound was synthesized and purified according to the procedure described by Allen et al. 13 who first measured the crystal NLO properties. Next, a crude material was purified by sublimation under vacuum ("instabilities at the melt temperature preclude zone refining”, according to ref. 13). Sublimed material has been extracted from the central part of a sublimation tube. Single crystals of DCNP were grown in the dark from $100 \mathrm{~cm}^{3}$ toluene DCNP solution, prepared at $+40{ }^{\circ} \mathrm{C}$, and using a slow cooling rate of approximately $0.1{ }^{\circ} \mathrm{C}$ per day. The resulting single crystal plates were formed spontaneously at the vessel edges when the temperature of the solution approached room temperature. Some crystals have thicknesses between 0.5 and $1 \mathrm{~mm}$ and areas between 5 and $10 \mathrm{~mm}^{2}$. The crystals used in this work for luminescence studies were in the form of parallelepipeds with approximate dimensions $10 \times 5 \times$ $2.5 \mathrm{~mm}^{3}$. The crystallographic $b$-axis is perpendicular to the plate face. The optical quality of the crystals obtained by us has been checked using a polarization microscope on the (010) natural face of the single crystal showing that the principal $z$-axis of the refractive index ellipsoid lies at an angle of $26^{\circ}$ with respect to the crystallographic $c$-axis. Along this $z$-axis long molecular axes are aligned.

\subsection{Spectroscopic measurements}

Spectroscopic studies of DCNP monomer molecules in a Shpol'skii, polycrystalline matrix of $n$-nonane were performed at $5 \mathrm{~K}$. The solution of DCNP in liquid $n$-nonane (concentrations $10^{-5}-10^{-4} \mathrm{M}$ ) was poured into a small cylinder-shaped fused silica cuvette and quickly cooled in liquid nitrogen (77 K) to prevent aggregation. Next, a sample holder with a mounted cuvette was inserted into an optical, liquid helium cryostat.

Absorption spectra of the crystal were recorded in a singlebeam configuration. In this case the light of a xenon lamp transmitted through the sample was dispersed using a McPherson 207 monochromator and detected using an EMI96659 photomultiplier operating in photon counting mode.

Fluorescence spectra were recorded at the right angle with respect to excitation, the emission light was dispersed using a McPherson 207 monochromator and detected either using an EMI96659 photomultiplier or using an EMCCD camera (Andor, model DU420A-BR-CD) cooled to $183 \mathrm{~K}$. As an excitation source we used light emitted by either a cw $532 \mathrm{~nm}(10 \mathrm{~mW})$ or $445 \mathrm{~nm}$ (20 $\mathrm{mW}$ ) solid state lasers. Alternatively we used a Lambda Physics FL1001 dye laser pumped using a pulsed LPX100 excimer laser $\left(\lambda_{\text {exc }}=308 \mathrm{~nm}\right)$ which when operated with the Coumarin 102 dye was scanned within the spectral range between 470 and $500 \mathrm{~nm}$ and with Coumarin 153 between 530 and $605 \mathrm{~nm}$, respectively. In the case of pulsed excitation, fluorescence signals were detected by the sampling technique using a photomultiplier and a Stanford Research SR259 boxcar averager.

The Raman scattering experiment was performed at $295 \mathrm{~K}$ using for excitation a cw Krypton laser operating at $\lambda_{\mathrm{R}}=676.4 \mathrm{~nm}$. The Raman spectrum was recorded using a Jobin Yvon T64000 spectrograph equipped with a CCD camera cooled by liquid nitrogen as a detector. The $676.4 \mathrm{~nm}$ light was weakly absorbed by a DCNP crystal, leading to weak fluorescence, and this fluorescence background was numerically subtracted from the detected Raman spectrum.

\subsection{Emission kinetics measurements}

Fluorescence kinetics studies were done with the aid of the "time correlated" single photon counting technique (in the inverted time mode). Excitation pulses were provided either by the second harmonics of a mode-locked Coherent's Mira-HP picosecond laser pumped by a Verdi18 laser or by the picosecond pulses from an APE Optical Parametric Oscillator pumped by a Mira-HP/Verdi18 system. The original repetition rate of a Mira-HP laser, $80 \mathrm{MHz}$, was reduced to $2 \mathrm{MHz}$ with the aid of an APE Pulse Selector. The fluorescence photons were dispersed with a McPherson 207 monochromator and the fluorescence decay curves were monitored with a HMP-100-50 hybrid detector and a SPC-150 module inserted into a PC, both from Becker\&Hickl GmbH. Fluorescence decay times were determined by using the deconvolution program, which iteratively fitted the theoretical curve to the experimental decay with the aid of the least-squares Levenberg-Marquardt algorithm.

\section{Conclusions}

We studied absorption and fluorescence spectra of a noncentrosymmetric highly polar single crystal of DCNP at a temperature of $5 \mathrm{~K}$. The bottom of the excitonic band was assigned to lay at $\sim 18115 \mathrm{~cm}^{-1}(552 \mathrm{~nm})$. We argue that the fluorescence emission, which spans between 552 and $575 \mathrm{~nm}$, 
has an excitonic origin. Such a conclusion is confirmed by the vibrational structure of fluorescence closely resembling that observed in the Raman scattering spectrum of DCNP crystals as well as by the very short, subnanosecond decay time of fluorescence, being a fingerprint of exciton migration and trapping at deep traps. Fluorescence decay times from the trap states in a DCNP crystal and that for a DCNP monomer in a Shpol'skii matrix are much longer, approaching about 5 nanoseconds.

An important result of the work is to convince that the vibrational structure of the Raman spectrum (recorded under ambient conditions) can be successfully used to reproduce the vibrational structure of the fluorescence spectrum recorded at the cryogenic temperature $(5 \mathrm{~K})$. Good matching of the Raman spectrum and the fluorescence spectrum of the deep (at $5 \mathrm{~K}$ ) trap (trap with the depth of $\sim 875 \mathrm{~cm}^{-1}$, and emission onset at $582 \mathrm{~nm}$ ) argues that this trap is a kind of structural defect and does not correspond to a chemical impurity.

Finally, let us add that the present contribution is dedicated to the optical study of a DCNP crystal at $5 \mathrm{~K}$. The optical studies of the crystal at elevated temperatures, where the deep traps play a dominant role and thus their origin is of main importance, will be considered in a forthcoming elaboration.

\section{Acknowledgements}

This work was financially supported by the Polish National Science Centre, Dec-2011/03/B/ST5/01021 and the grant no. 2012/04/A/ST2/00100. We also wish to thank Dr T. Misiaszek from the Wroclaw University of Technology for measurement of the Raman spectrum in a DCNP crystal.

\section{Notes and references}

1 A. E. Rashad, A. H. Shamroukh, M. I. Hegab and H. M. Awad, Acta Chim. Slov., 2005, 52, 429-434.

2 B. A. Bhat, K. L. Dhar, A. K. Saxena and M. Shanmugavel, Bioorg. Med. Chem., 2005, 15, 3177-3180.

3 E. M. Sharshira and N. M. Hamada, Molecules, 2011, 16, 7736-7745.

4 C. W. Dirk, H. E. Katz, M. L. Shilling and L. A. King, Chem. Mater., 1990, 2, 700-705.
5 Y. Dwivedi, L. de Boni, P. J. Gonçalves, L. M. Mairink, R. Menegatti, T. L. Fonseca and S. C. Zilio, Spectrochim. Acta, Part A, 2013, 105, 483-487.

6 R. D. Miller, C. R. Moylan, O. Reiser and C. A. Walsh, Chem. Mater., 1993, 5, 625-632.

7 S. Tabak, I. I. Grandberg and A. N. Kosrt, Tetrahedron, 1966, 22, 2703-2710.

8 S. Chatterjee, A. Mukharjee, K. K. Mahalanabis and S. C. Bhattacharya, J. Surf. Sci. Technol., 2008, 24, 195-205.

9 J. M. Cole, C. C. Wilson and J. A. K. Howard, Mol. Cryst. Liq. Cryst. Sci. Technol., Sect. B, 2000, 25, 265-270.

10 A. Radi, S. Radi and B. Hammouti, J. Mater. Environ. Sci., 2010, 1, 96.

11 K. A. Vishnumurthy, A. V. Adhikari, M. S. Sunitha and R. Philip, AIP Conf. Proc., 2011, 1391, 652.

12 S. Allen, T. D. McLean, P. F. Gordon, B. D. Bothwell, M. B. Hursthouse and S. A. Karaulov, J. Appl. Phys., 1988, 64, 2583-2590.

13 J. M. Cole, C. C. Wilson, J. A. K. Howard and F. R. Cruickshank, Acta Crystallogr., Sect. B: Struct. Sci., 2000, 56, 1085-1093.

14 A. Miniewicz, K. Palewska, L. Sznitko and J. Lipinski, J. Phys. Chem. A, 2011, 115, 10689-10697.

15 L. Sznitko, J. Mysliwiec, K. Parafiniuk, A. Szukalski, K. Palewska, S. Bartkiewicz and A. Miniewicz, Chem. Phys. Lett., 2011, 512, 247-250; A. Miniewicz, K. Palewska, P. Karpinski, L. Sznitko and M. Zielinski, Nanobiosystems: Processing, Characterization, and Applications V, Proc. SPIE, 2012, 8464, 84640O.

16 W. Shi, C. Fang, Z. Xu, Q. Pan, Q. Gu, D. Xu, H. Wei and J. Yu, Solid State Commun., 2000, 113, 483-487.

17 W. Shi, C. Fang, Q. Pan, X. Sun, Q. Gu, D. Xu, H. Wei and J. Yu, Chin. Phys. Lett., 2000, 17, 22-24.

18 A.-M. Manea, I. Rau, A. Tane, F. Kajzar, L. Sznitko and A. Miniewicz, Opt. Mater., 2013, 36, 69-74.

19 S. N. Black, R. J. Davey, P. R. Morley, P. Halfpenny, E. E. A. Shepherd and J. N. Sherwood, J. Mater. Chem., 1993, 3, 129-132.

20 M. Pope and C. E. Swenberg, Electronic processes in organic crystals, Clarendon Press, 1982.

21 M. R. Philpott, J. Chem. Phys., 1971, 55, 2039-2054. 